\title{
Some Mapping Properties of Representing Measures (*).
}

\author{
Russell Bilyeu - Paul Lewis (Denton, Texas, U.S.A.)
}

Summary. - This paper studies relationships between operators on continuous function spaces and properties of associated vector measures given by Riesz Representation Theorems.

\section{1. - Introduction and definitions.}

Suppose that each of $E$ and $F$ is a $B$-space (= Banach space), $H$ is a compact Hausdorff space, and $C(H, E)$ is the $B$-space (re sup norm) of all continuous $E$-valued functions on $H$. We will be interested in operators $L: C(H, E) \rightarrow F$ and representing measures $m: \Sigma \rightarrow B(E, F)$, where $B(E, F)$ is the $B$-space of all operators from $E$ into $F$ and $\Sigma$ is the Borel $\sigma$-algebra of subsets of $H$. A finitely additive set function $m: \Sigma \rightarrow B\left(E, F^{* *}\right)$ is called a representing measure if $m$ has finite semivariation and $\left|m_{z}\right|$ is a regular Borel measure for each $z \in F_{1}^{*}$ (= closed unit ball in the dual of $F$ ). The Riesz Representation Theorem in this setting asserts that to each operator $L: C(H, E) \rightarrow F$ there may be associated a unique representing measure $m: \Sigma \rightarrow B\left(E, F^{* *}\right)$ so that $L(f)=\int_{H} f d m$ and $\|L\|=\tilde{m}(H)$, where $\tilde{m}$ denotes the semivariation; this association is denoted by $L \leftrightarrow m$. The reader may consult Brooks and Lewis [4] and Dinculeanu [8] for a detailed discussion of this setting. In particular, $\xi_{A}$ will denote the characteristic function of a set $A, S(\Sigma)$ will denote the scalar valued simple functions defined over $\Sigma$, and $U(\Sigma)$ will be the uniform closure of $S(\Sigma)$. The spaces $S_{E}(\Sigma)$ and $U_{E}(\Sigma)$ are defined analogously for $E$-valued functions. The reader should note that if $m \leftrightarrow L$, then $m(A) x==I^{* *}\left(\xi_{A} x\right)$. The majority of our results will be concerned with relating properties of the operator $L$ to properties of its representing measures.

The classes of operators that we will discuss are the compact, weakly compact, absolutely $p$-summing, nuclear, quasinuclear, unconditionally converging, completely continuous, strictly singular, and strongly bounded. An operator $T: E \rightarrow F$ is said to be (weakly) compact if it maps bounded sets in $E$ into conditionally (weakly) compact sets in $F$, and $T$ is said to be absolutely $p$-summing (a.p.s.) if it maps wealkly $p$-summable (w.p.s.) series in $E$ into absolutely $p$-summable series in $F$. Equivalently, $T$ is a.p.s. if there is a constant $\varrho \geqslant 0$ so that if $x_{1}, \ldots, x_{k}$ is a finite set from $E$, then

$$
\left(\sum_{i=1}^{k}\left\|T\left(x_{i}\right)\right\|^{p}\right)^{1 / p} \leqslant \varrho \sup \left\{\left(\sum\left|x^{*}\left(x_{i}\right)\right|^{p}\right)^{1 / p}: x^{*} \in E_{1}^{*}\right\} .
$$

(*) Entrata in Redazione il 2 aprile 1975 . 
If $T$ is a.p.s., then the infimum of all numbers $\varrho \geqslant 0$ satisfying (*) is called the absolutely $p$-summing norm of $T$ and is denoted by $\pi_{p}(T)$; and

$$
\sup \left\{\left(\sum_{i=1}^{k}\left|x^{*}\left(x_{i}\right)\right|^{p}\right)^{1 / p}: x^{*} \in E_{1}^{*}\right\}
$$

is denoted by $\varepsilon_{y}\left(x_{i}\right)_{i=1}^{k}$. The mapping $T$ is said to be nuclear if there is a sequence $\left(a_{n}\right) \subset E^{*}$ and a sequence $\left(b_{n}\right) \subset F$ so that

$$
\sum_{n=1}^{\infty}\left\|a_{n}\right\|\left\|b_{n n}\right\|<\infty \quad \text { and } \quad T(x)=\sum_{n=1}^{\infty} a_{n}(x) b_{n} \quad \text { for each } x \in E
$$

Also, $T$ is said to be unconditionally converging (u.c.) if $T$ maps weakly unconditionally converging (w.u.e. $\equiv$ w.1.s.) series in $E$ into unconditionally converging series in $F, T$ is said to be completely continuous if $T$ maps weak Cauchy sequences in $E$ into norm convergent sequences in $F$, and $T$ is said to be strictly singular if $T$ does not have a bounded inverse on any infinite dimensional subspace of $E$.

\section{2. - Absolutely $p$-summing operators and the $\pi_{p}$-variation.}

We begin this section with a lemma from Brooks and LewIs [5]; we include a proof for completeness. The notation $E \otimes_{\lambda} F$ is used for the least crossnorm completion of $E \otimes F$.

2.1 LeMma - If $\sum x_{i}$ is weakly $p$-summable in $E$ and $\left(y_{i}\right) \subset F_{1}$ (= closed unit ball of $F$ ), then $\sum x_{i} \otimes y_{i}$ is weakly $p$-summable in $E \otimes_{\lambda} F$.

Proof. - Suppose that $\sum x_{i}$ is w.p.s. in $X$ and that there is a sequence $\left(y_{i}\right) \subset Y_{1}$ so that $\sum x_{i} \otimes y_{i}$ is not w.p.s. in $X \otimes \otimes_{\lambda} Y$. Thus there is an integer $N$ and $\gamma \in$ $\in\left(X \otimes \otimes_{n} Y_{1}^{*}\right)$ with $\left(\sum_{1}^{N}\left|\gamma\left(x_{i} \otimes y_{i}\right)\right|^{p}\right)^{1 / p}>\varepsilon_{p}\left(x_{i}\right)$. Thus there is an element $w=\left(w_{i}\right)$ $\in\left(l^{r}\right)_{1}$ so that $\sum_{1}^{N} \gamma\left(x_{i} \otimes y_{i}\right) w_{i}>\varepsilon_{y}\left(x_{i}\right)$. Therefore,

$$
\gamma\left(\sum_{1}^{N} x_{i} \otimes w_{i} y_{i}\right)>\varepsilon_{y}\left(x_{i}\right)
$$

and

$$
\sum^{N}\left\|x_{i} \otimes w_{i} y_{i}\right\|_{\lambda}>\varepsilon_{p}\left(x_{i}\right)
$$


But then there is an $f \in X_{1}^{*}$ so that $\left\|\sum f\left(x_{i}\right) w_{i} y_{i}\right\|>\varepsilon_{p}\left(x_{i}\right)$. Consequently,

$$
\begin{aligned}
\varepsilon_{y}\left(x_{i}\right) & <\sum_{1}^{N}\left\|f\left(x_{i}\right) w_{i} y_{i}\right\| \\
& \leqslant \sum_{1}^{N}\left|f\left(x_{i}\right) w_{i}\right| \leqslant\left(\sum_{i=1}^{\infty}\left|f\left(x_{i}\right)\right|^{p}\right)^{1 / p}\left(\sum\left|w_{i}\right|^{q}\right)^{1 / q} \\
& \leqslant\left(\sum\left|f\left(x_{i}\right)\right|^{p}\right)^{1 / p} \leqslant \varepsilon_{y}\left(x_{i}\right),
\end{aligned}
$$

and we have a contradiction.

As an application of the lemma, we obtain the following result.

2.2 Poposition. - Suppose that $L: E \otimes_{\lambda} F \rightarrow G$ is an operator where $G$ is a $B$-space, and that $L_{1}: E \rightarrow B(F, G)$ is the naturally induced operator. Then

(i) $L_{1}$ is unconditionally converging if $L$ is unconditionally converging;

(ii) $L_{1}$ is absolutely $p$-summing if $L$ is absolutely $p$-summing.

Proof. - (i) Suppose that $\sum x_{i}$ is w.u.c. in $E$ and that $\left(n_{i}\right)$ is a permutation of $\boldsymbol{N}$ so that $\sum_{i=1}^{\infty} L_{1}\left(x_{n_{i}}\right)$ does not converge. Then choose a sequence $\left(y_{i}\right) \subset F_{1}$ so that $\sum\left\|L_{1}\left(x_{n_{i}}\right)-L\left(x_{n_{i}} \otimes y_{i}\right)\right\|<1$. Since $\left(y_{i}\right)$ is bounded, by Lemma $2.1, \sum x_{n_{i}} \otimes y_{i}$ is w.u.c. in $E \otimes_{\lambda} F$; consequently, $\sum L\left(x_{n_{i}} \otimes y_{i}\right)$ is u.c. But then $\sum L_{1}\left(x_{n_{i}}\right)$ must be u.c., a contradiction. Thus if $L$ is u.c., if follows that $L_{1}$ is u.c.

(ii) The proof of (ii) is similar; the details will be omitted.

The preceding lemma and proposition generalize some recent results of SWARTz [22]. In particular, SWARTz showed that if $L: C(H, E) \rightarrow F$ is absolutely summing, then $L_{1}: C(H) \rightarrow B(E, F)$ is absolutely summing. Since $O(H, E) \cong C(H) \otimes \otimes_{2} E$, the relationship of this fact to Proposition 2.2 is clear. We mention that one may also employ a method similar to the one used in [21] to show that if $L: O(H, E) \rightarrow F$ is absolutely $p$-summing, then $L_{1}: C(H) \rightarrow B(E, F)$ is absolutely $p$-summing. A key ingredient needed in this approach is the following lemma.

2.3 Lemma. - Suppose that $f_{1}, \ldots, f_{k} \in C(H)$.

(i) If $\varepsilon_{p}\left(f_{i}\right) \leqslant 1$ then $\varepsilon_{p}\left(\left|f_{i}\right|\right) \leqslant 2$.

(ii) If $\varepsilon_{p}\left(f_{i}\right)=1$, then $\left\|\sum_{i=1}^{k}\left|f_{i}\right|^{p}\right\|_{\infty}=1$.

Proof. - Suppose that $f_{1}, \ldots, f_{k}$ satisfy the hypothesis of the lemma, $U=C(H)_{1}$, and set $U_{+}^{0}=\left\{\mu \in U_{0}: \mu \geqslant 0\right\}$, i.e., $D_{0}$ is the polar of $U$ in $C(H)^{*}$, and $U_{+}^{0}$ consists of all non-negative regular Borel measures in $U^{0}$. Then $U_{+}^{0}$ is the $w^{*}$-closure of the extreme points of $U_{+}^{0}$. But an extreme point of $U_{+}^{0}$ is either a point mass $\delta_{t}, t \in B$, 
or the zero measure. Now suppose that $\varphi=\sum_{i=1}^{n} \alpha_{i} \delta_{t_{i}}, \sum \alpha_{i}>0, \sum \alpha_{i} \leqslant 1$. Then,

$$
\begin{aligned}
& \left(\sum_{i=1}^{k_{k}} \mid\left(\left.\sum_{j=1}^{n} \alpha_{j} \delta_{t_{j}}\left|f_{i}\right|\right|^{p}\right)^{1 / p}\right. \\
= & \left(\sum_{i=1}^{n}\left(\sum_{j=1}^{k} \alpha_{j} \delta_{t_{j}}\right)\left|f_{i}\right|^{p}\right)^{1 / p} \\
\leqslant & \left(\sum_{i=1}^{n}\left(\sum_{j=1}^{k}\left(\alpha_{j} \delta_{t_{j}}\left|f_{i}\right|\right)^{p}\right)^{1 / p}\right. \\
= & \sum_{j=1}^{n} \alpha_{j}\left(\sum\left|\delta_{t_{j}}\left(f_{i}\right)\right| p\right)^{1 / p} \\
\leqslant & \sum_{j=1}^{n} \alpha_{j} \leqslant 1 .
\end{aligned}
$$

As a consequence, if $\varphi \in U_{+}^{0}$, then $\left(\sum \varphi\left(\left|f_{i}\right|\right)^{p}\right)^{p / 1} \leqslant 1$. But if $\varphi \in U_{0}$, then $\varphi=\varphi^{+}-\varphi^{-}$, where each of $\varphi^{+}$and $\varphi^{-}$belongs to $U_{+}^{0}$. Therefore

$$
\begin{aligned}
& \left(\sum\left|\varphi\left(\left|f_{i}\right|\right)\right|^{p}\right)^{1 / p} \\
= & \left(\sum\left|\left(\varphi^{+}-\varphi^{-}\right)\right| f_{i}||^{p}\right)^{1 / p} \\
\leqslant & \left(\sum\left(\varphi^{+}\left|f_{i}\right|+\varphi^{-}\left|f_{i}\right|\right)^{p}\right)^{1 / p} \\
\leqslant & \left(\sum\left(\varphi^{+}\left|f_{i}\right|\right)^{p}\right)^{1 / p}+\left(\sum\left(\varphi^{-}\left|f_{i}\right|\right)^{p}\right)^{1 / p} \leqslant 2,
\end{aligned}
$$

and the proof of (i) is finished.

The proof of (ii) is analogous to that of (i) and will be omitted.

A consequence of (i) is that if $\sum f_{i}$ is w.p.s. in $C(H)$, then $\sum\left|f_{i}\right|$ is w.p.s.

It was also shown in [22] that an operator

$$
m \leftrightarrow L: C(H, E) \rightarrow F
$$

is absolutely summing if and only if $m$ has finite $\pi$-variation. (See Definition 2.4 below.) However, the situation is more delicate for $p>1$.

2.4 Definition. - Let $m \leftrightarrow L: C(H, E) \rightarrow F$ be an absolutely $p$-summing operator. Then By Theorem 4.1. infra, $m(A): E \rightarrow F$ is a.p.s. for each $A \in \Sigma$. Define $\pi_{p}(m)$ to be $\sup _{\pi(H)}\left\{\sum \pi_{p}\left(m\left(A_{i}\right)\right)\right\}$, where $\pi(H)$ denotes the collection of all finite disjoint $\Sigma$-partitions of $H$. Similarly, define $\pi_{p}^{p}(m)$ to be $\sup \left\{\sum \pi_{p}\left(m\left(A_{i}\right)^{p}\right)^{1 / p}\right\}$.

2.5 THEorem. - If $m \leftrightarrow L: C(H, E) \rightarrow F$ is absolutely $p$-summing, then $\pi_{p}^{p}(m)<\infty$. Conversely, if $\pi_{p}(m)<\infty$, then $L$ is absolutely $p$-summing. 
Proof. - Suppose that $L$ is a.p.s., let $\varepsilon>0$, let $A_{1}, \ldots, A_{k}$ be a partition of $H$, and let $\left(x_{n i}\right)_{i}, n=1, \ldots, k$ be a collection of finite subsets of $E$ so that $\varepsilon_{p}\left(x_{n i}\right) \leqslant 1$ for each $n$ and

$$
\left(\sum_{n=1}^{n} \pi_{p}\left(m\left(A_{n}\right)^{p}\right)^{1 / p}<\left(\sum_{n} \sum_{i}\left\|m\left(A_{n}\right)\left(x_{n_{i}}\right)\right\|^{p}\right)^{1 / p}+\varepsilon\right.
$$

Then,

$$
\left(\sum_{n} \sum_{i}\left\|m\left(A_{n}\right) x_{n i}\right\|^{p}\right)^{1 / p}=\left(\sum_{n} \sum_{i}\left\|L^{* *}\left(\xi_{A_{n}} x_{n i}\right)\right\|^{p}\right)^{1 / p} \leqslant \pi_{p}\left(L^{* *}\right) \varepsilon_{p}\left(\xi_{A_{n}} x_{n i}\right)_{n, i} .
$$

(Recall that $L^{* *}$ is absolutely $p$-summing).

Then suppose that $v \in U_{Z}(\Sigma)^{*},\|v\| \leqslant 1$, and note that

$$
\left(\sum_{n} \sum_{i}\left|\nu\left(\xi_{A_{n}} x_{n i}\right)\right|^{p}\right)^{1 / p} \leqslant \sum_{n}\left(\sum_{i}\left|v\left(\xi_{A_{n}} x_{n i}\right)\right|^{p}\right)^{1 / p}
$$

Define $\left(\nu, \xi_{A}\right): E \rightarrow \mathbb{R}$ by $\left(\nu, \xi_{A}\right) x=v\left(\xi_{A} x\right) ;$ consequently, $\left(\nu, \xi_{A}\right) \in E^{*}$, and $\left\|\left(v, \xi_{A}\right)\right\|_{E^{*}} \leqslant$ $\leqslant|v|(A)$. Thus

$$
\sum_{n}\left(\sum_{i}\left|v\left(\xi_{A_{n}} x_{n i}\right)\right|^{p}\right)^{1 / s} \leqslant \sum_{n}\left(\nu, \xi_{A_{n}}\right) \| \varepsilon_{p}\left(x_{n_{i}}\right)_{i} \leqslant \sum_{n}|\nu|\left(A_{n}\right) \leqslant 1
$$

Therefore $\pi_{p}^{p}(m) \leqslant \pi_{p}\left(L^{* *}\right)$. And PIETscr [17] has shown that $\pi_{y}\left(L^{* *}\right)=\pi_{p}(L)$; hence $\pi_{p}^{p}(m) \leqslant \pi_{p}(L)$.

Conversely, suppose that $\pi_{p}(m)<\infty$, and let $\varphi_{1}, \ldots, \varphi_{k} \in \mathbb{S}_{E}(\Sigma)$ so that $\varepsilon_{p}\left(\varphi_{i}\right)_{i=1}^{k} \leqslant 1$. Without loss of generality, suppose that

$$
\varphi_{i}=\sum_{n=1}^{t} \xi_{A n} x_{n i}
$$

for each $i$ and that no $A_{n}$ is empty. Then

$$
\begin{aligned}
\left(\sum_{i}\left\|L^{* *}\left(\varphi_{i}\right)\right\|^{p}\right)^{1 / p} & =\left(\sum_{i}\left\|\sum_{n} m\left(A_{n}\right) x_{n i}\right\|^{p}\right)^{1 / p} \\
& \leqslant\left(\sum_{i} \sum_{n}\left\|m\left(A_{n}\right) x_{n i}\right\|^{p}\right)^{1 / p} \\
& \leqslant\left(\sum_{i} \sum_{n}\left\|m\left(A_{n}\right) x_{i n}\right\|^{p}\right)^{1 / p}
\end{aligned}
$$

Also, since $\varepsilon_{p}\left(\varphi_{i}\right)_{i=1}^{k} \leqslant 1$, it follows that $\varepsilon_{p}\left(x_{n i}\right)_{i} \leqslant 1$ for each $n$. Therefore,

$$
\sum_{n}\left(\sum_{i}\left\|m\left(A_{n}\right) x_{n i}\right\|^{p}\right)^{1 / p} \leqslant \sum_{n} \pi_{p}\left(m\left(A_{n}\right)\right) \leqslant \pi_{p}(m) .
$$


We remark that it is possible for $L$ to be a.p.s. and $\pi_{p}(m)$ to be unbounded. For example, let

$$
\mathfrak{J}: C\left[0, \frac{\pi^{2}}{6}\right] \rightarrow L^{2}\left[0, \frac{\pi^{2}}{6}\right]
$$

be the identity injection; then $\mathfrak{J} \leftrightarrow \mu$, where

$$
\mu(A)=\xi_{\Delta} \in B\left(\mathbb{R}, L^{2}\left[0, \frac{\pi^{2}}{6}\right]\right) \simeq L^{2}\left[0, \frac{\pi^{2}}{6}\right] .
$$

Let $\left(A_{n}\right)$ be a sequence of disjoint Borel subsets of $\left(0, \pi^{2} / 6\right]$ so that $\lambda\left(A_{n}\right)=1 / n^{2}$, where $\lambda$ is Lebesgue measure. Then certainly $\pi_{p}\left(\mu\left(A_{n}\right)\right) \geqslant\left\|\mu\left(A_{n}\right)\right\|=\left(\lambda\left(A_{n}\right)\right)^{\frac{1}{2}}=1 / n$ and therefore $\pi_{p}(m)=\infty$.

\section{3. - Strongly bounded operators and $c_{0}$.}

The absolutely $p$-summing operators of the preceding section form a subelass of the strongly bounded operators. (See the opening remarks of $\S 4$.)

3.1. Definition. - An operator $m \leftrightarrow L: C(H, E) \rightarrow F$ is said to be strongly bounded (=s-bounded) provided that if $\left(A_{i}\right)$ is a disjoint sequence from $\Sigma$, then $\tilde{m}\left(A_{i}\right) \rightarrow 0$. We speak of an operator and its representing measure being $s$-bounded interchangeably.

The following lemma, whose proof can be found in [4], presents alternate characterizations of $s$-bounded representing measures.

3.2 Lemma. - If $m: \Sigma \rightarrow B(E, F)$ is a representing measure, then the following are equivalent.

(i) $m$ is $s$-bounded;

(ii) if $A_{n} \searrow \phi$, then $\tilde{m}\left(A_{n}\right) \searrow 0$;

(iii) $\left\{\left|m_{z}\right|: z \in F_{1}^{*}\right\}$ is conditionally weakly compact in $\operatorname{rca}(\Sigma)$;

(iv) $m\left(A_{i}\right) x_{i}$ converges in $F$ for each disjoint sequence $\left(A_{i}\right)$ and each sequence $\left(x_{i}\right) \subset E_{1}$.

The class of strongly bounded operators is in a sense the natural extension of weakly compact operators $T: C(H) \rightarrow F$ to the $C(H, E)$-setting. For a discussion of strongly bounded operators in the $C(H, E)$-setting, see Brooks and LEWIs [4].

Another way in which the strongly bounded operators may be thought to as a generalization of the weakly compact operators can be revealed in connection with the following theorem of Pelczynski.

THEonem [15]. - If the operator $L: C(H) \rightarrow F$ is not weakly compact, then $C(H)$ contains an isometric copy $Y$ of $\varepsilon_{0}$ so that the restriction of $L$ to $Y$ is an isomorphism. 
While the direct generalization of the above theorem is false for non weakly compact operators defined on vector-valued continuous function spaces, using the techniques of [15] and the following lemma from Rosenthal [19], we establish a result analogous to the above theorem.

3.3 Lemira [19]. - Let $\int$ be the power algebra of the natural numbers, let $0 \leqslant \mu_{i} \in$ $\in b f a(\mathfrak{T})$, and suppose that $\left\|\mu_{i}\right\| \leqslant 1$ for $i=1,2, \ldots$. If $\left(A_{i}\right)$ is a disjoint sequence from $\mathfrak{T}$ and $\delta>0$, then there is a subsequence $n_{i}$ so that

$$
\mu_{n_{i}}\left(\bigcup_{j \neq i} A_{n_{i}}\right)<\delta, \quad i=1,2, \ldots
$$

The correspondence $m \leftrightarrow L$ referred to in the following theorem is given by Theorem II.9.1 in Dinculeand [8].

3.4 THEOREM. - Suppose that $\Sigma$ is an algebra of subsets of an abstract set $W$, $S_{H}(\Sigma)$ is the space of $E$-valued simple functions over $\Sigma, U_{E}(\Sigma)$ is the uniform elosure of $S_{E}(\Sigma)$, and $m \leftrightarrow L: U_{E}(\Sigma) \rightarrow F$ is an operator which is not strongly bounded. Then $U_{E}(\Sigma)$ contains an isometric copy $Y$ of $e_{0}$ on which $L$ is an isomorphism.

Proof. - Let $S(\Sigma)$ be the scalar valued $\Sigma$-simple functions and let $O(\Sigma)$ be the uniform closure of $S(\Sigma)$. Then

$$
U_{E}(\Sigma) \cong U(\Sigma) \otimes \lambda E
$$

Furthermore, by the Stone Representation Theorem [8], there is a totally disconnected compact Hausdorfi space $S_{1}$ so that $\Sigma$ is isomorphic as a Boolean algebra to the collection of clopen subsets of $S_{1}$, and consequently $U(\Sigma) \cong O\left(S_{1}\right)$. Thus

$$
U_{E}(\Sigma) \cong C\left(S_{1}\right) \otimes \lambda E \cong C\left(S_{1}, E\right)
$$

and the naturally induced operator $L: O\left(\mathcal{S}_{1}, E\right) \rightarrow E$ is not $s$-bounded.

Therefore if we continue to denote the representing measure of $L$ by $m$, then $S=\left\{\left|m_{z}\right|: z \in F_{1}^{*}\right\}$ is not conditionally weakly compact. Combining results from Peldzynski [15] and Lemma 3.3, we obtain

(a) $\delta>0$,

(b) a disjoint sequence $O_{i}$ of open sets,

(c) a sequence $\left(z_{i}\right) \subset F_{1}^{*}$,

and

(d) a sequence $\left(g_{i}\right) \subset C(H, E)$ so that $\left\|g_{i}\right\|=1$ for each $i$ and support $\left(g_{i}\right) \subset O_{i}$ 
which satisfy

(e) $\left|m_{z_{i}}\right|\left(\bigcup_{k \neq i} O_{k}\right)<\delta / 2$

and

(f) $\left|L^{*}\left(z_{i}\right)\left(g_{i}\right)\right|=\left|\int g_{i} d m_{z_{i}}\right|>\delta, i=1,2, \ldots$

From $(d)$ it follows that if $Y=\overline{\operatorname{span}\left(g_{i}\right)}$, then $Y=c_{0}$. And from $(e)$ and $(f)$ it follows that $\left.L\right|_{Y}$ is an isomorphism. Pulling back through the isometries, the theorem follows.

Joe DIEsTeL and Jerry UHL have obtained results similar to the preceding theorem (private communication).

We also note that if $L: C(H, E) \rightarrow F$ is not $s$-bounded, then there may be many isometric copies of $c_{0}$ in $C(H, E)$ on which $L$ is not an isomorphism. To see this, let $H, E$, and $F$ be such that there is an operator $m \leftrightarrow L: O(H, E) \rightarrow F$ with $m$ countably additive and not $s$-bounded. From the proof of Theorem 3.4 it follows that $O(H)$ must be infinite dimensional; hence $O(H)$ contains an isometric copy $Y$ of $c_{0}$. Thus $O(H) \cdot x$ contains an isometric copy of $Y \cdot x$ of $c_{0}$ for each vector $x \in E$ so that $\|x\|>0$. But $L: O(H) x \rightarrow F$ is weakly compact since the representing measure for the restriction of $L$ to $C(H) x$ is countably additive. (If $X$ is a finite dimensional $B$-space and $Y$ is any $B$-space, then a countably additive representing measure is strongly bounded.) Therefore $\left.L\right|_{Y x}: Y x \rightarrow F$ cannot be an isomorphism.

REMARK. - A recent result by DIESTEL and FAIREs [7] characterizing strongly additive measures, together with Theorem 4.2 of [4], makes possible the following characterization of nonstrongly additive operators on $U_{E}(\Sigma)$-spaces, where $\Sigma$ is a $\sigma$-algebra. (An operator $m \leftrightarrow L: U_{E}(\Sigma) \rightarrow F$ is strongly additive if $m\left(A_{i}\right) \rightarrow 0$ whenever $\left(A_{i}\right)$ is a disjoint sequence from $\Sigma$.) If $E$ and $F$ are Banach spaces, then an operator $m \leftrightarrow L: U_{E}(\Sigma) \rightarrow E$ is not strongly additive if and only if there is an isometrie copy $W$ of $l^{\infty}$ in $U(\Sigma)$ so that $L_{\left.1\right|_{W}}$ is an isomorphism, where $L_{1}: U(\Sigma) \rightarrow B(E, F)$ is defined by $L_{1}(f)=\int f d m$.

Our next result deals with linear best approximation projections onto $c_{0}$. Specifically, if $E$ is a separable subspace of $m\left(=l^{\infty}=\right.$ bounded continuous functions on $\mathbb{N}$ ) containing $c_{0}$, such that, for each $x \in E, P(x)$ is a best approximation to $x$ from $c_{0}$. This fact seems to have gone unnoticed even though the essence of the proof is well known, having been discovered by KöTHE [12], elucidated by GoLDBERG [10], and rediscovered by VEECH [23]. These authors conclude that there is a projection from $E$ onto $c_{0}$ having norm 2 , a fact originally announced by SoBczYK [20]. We are not able to get the stronger conclusion by Sobczyk's method.

The existence of such a projection $P$ is equivalent, via the complementary projection, to the existence of a norm one projection having $c_{0}$ as its kernel. This elementary fact is given by CHeney and PRICE [6], and also ANDERson [2], whose papers give some evidence of the rarity of linear best approximation operators.

Also equivalent to the existence of $P$ is the existence of a subspace $L$ of $E$, com- 
plementary to $o_{0}$, each element of which is orthogonal to each element of $c_{0}$. Each member of $L$ has the property that sup $\left|x_{n}\right|=\lim \sup \left|x_{n}\right|$.

3.5 THEoREM. - If $E$ is a separable subspace of $m$ containing $c_{0}$ then there exists a linear projection $P: E \rightarrow E$ such that $\|P\|=1$ and ker $P=c_{0}$.

Proof. - Let $H$ be the unit ball in the dual of $E$, equipped with the weak-star topology. Then $H$ is compact and metrizable. We select a metric $d$ for $H$. Let $\pi_{n}$ denote the $n$-th coordinate projection on $E$. Let $K$ consist of those members of $H$ which vanish on $c_{0}$. Then $K$ is compact, and there exists $\phi_{n}$ in $K$ such that $d\left(\pi_{n}, \phi_{n}\right)=$ $=d\left(\pi_{n}, K\right)$. The projection is defined by $P x=\left\{\phi_{n}(x)\right\}_{n=1}^{\infty}$ for $x$ in $E$.

Suppose $x-P x \notin c_{0}$. Then $d\left(\pi_{n}, \phi_{n}\right) \rightarrow 0$, and since $H$ is compact there is a subsequence of $\left\{\pi_{n}\right\}_{n=1}^{\infty}$ which converges to a point $\Psi \in H \backslash K$. This implies that $\Psi(x) \neq 0$ for some $x$ in $e_{0}$. But $\Psi(x)$ is the limit of a subsequence of $\left\{\pi_{n}(x)\right\}_{n=1}^{\infty}$; i.e., $\Psi(x)$ is the limit of a subsequence of $x$. Therefore $x-P x \in c_{0}$.

Certainly, then, $\operatorname{ker} P \subset c_{0}$. But also $c_{0} \subset \operatorname{ker} P$ because $\phi_{n} \in K$. That $P^{2}=P$ follows from

$$
P^{2} x-P x=P(P x-x) \in P\left(c_{0}\right)=\{0\} .
$$

Finally, $P$ is linear of norm one because each $\phi_{n}$ is linear of norm one.

\section{4. - Mapping properties of representing measures.}

In (21) SWARTZ showed that an unconditionally converging operator on $O(H, E)$ is strongly bounded. Thus it follows that every weakly compact, compact, nuclear, quasinuclear, absolutely $p$-summing, and completely continuous operator on $C(H, E)$ is $s$-bounded. Furthermore, HowARD [11] has shown that every strictly singular operater is unconditionally converging; therefore a strictly singular operator on $C(H, E)$ is $s$-bounded.

As a result of the strong boundedness, the reader should note that a representing: measure for a map in any of the classes of operators under consideration must map into $B(E, F)$, e.g. see Brooks and LewIs [4].

We note here that if $L: C(H, E) \rightarrow F$ is not strongly bounded, then Lemma 1 of PELCYNSKI [16] implies that there is a subspace $Y$ of $C(H, E)$ isomorphic to $e_{0}$ on which $L$ has a bounded inverse. However, this lemma does not give the isometry of Theorem 3.4 nor does it seem to reveal as much about the structure of these particular spaces.

The following theorem shows that many mapping properties of an operator $m \leftrightarrow L: O(H, E) \rightarrow F$ are shared by its representing measure $m$.

4.1 Theorem. - Let $m \leftrightarrow L: C(H, E) \rightarrow F$.

(i) If $L$ is weakly compact, then $m(A)$ is weakly compact for each $A \in \Sigma$. 
(ii) If $L$ is compact, then $m(A)$ is compact for each $A \in \Sigma$.

(iii) I $L$ is absolutely $p$-summing then $m(A)$ is absolutely $p$-summming for each $A \in \Sigma$.

(iv) If $L$ is unconditionally converging, then $m(A)$ is unconditionally converging for each $A \in \Sigma$.

(v) If $L$ is completely continuous, then $m(A)$ is completely continuous for each $A \in \Sigma$.

(vi) If $L$ is nuclear, then $m(A)$ is nuclear for each $A \in \Sigma$.

(vii) If $L$ is quasinuclear, then $m(A)$ is quasinuclear for each $A \in \Sigma$.

(viii) If $L$ is strictly singular, then $m(A)$ is strictly singular for each $A \in \Sigma$.

Proof. - (i) and (ii) follow from $\$ 4$ of Brooks and LEwIs [5].

(iii) Swartz [22] has shown that if $L$ is absolutely summing, then $m(A)$ is absolutely summing for each $A \in \Sigma$. Therefore suppose that $L$ is a.p.s. and that $1<p<\infty$. Puexscr [17] has shown that an operator $T$ is a.p.s. if and only if $T^{* * *}$ is a.p.s.; hence $L^{* *}: U_{E}(\Sigma) \rightarrow F$ is a.p.s. $\left(U_{E}(\Sigma)\right.$ is isometrically contained in $\left.C(H, E)^{* *}\right)$. Then, if $\sum x_{i}$ is w.p.s. in $E$ and $\xi_{A}$ is the characteristic function of $A \in \Sigma$, by Lemma 2.1 it follows that $\sum \xi_{A} x_{i}$ is w.p.s. in $U_{Z}(\Sigma) \cong U(\Sigma) \otimes_{\lambda} E$. Consequently, $\sum L^{* *}\left(\xi_{A} x_{i}\right)=\sum m(A) x_{i}$ is a.p.s., and it follows that $m(B)$ is a.p.s. for each $B \in \Sigma$.

(iv) Suppose that $L$ is u.c., $A \in \Sigma, \sum x_{i}$ is w.u.c., and $\sum m(A) x_{i}$ not u.c. in $F$. Without loss of generality, assume that $\varepsilon>0,\left(a_{k}, b_{k}\right)_{k=1}^{\infty}$ is a sequence of pairs of integers so that $a_{k}<b_{k}<a_{k+1}$ for all $k$, and

$$
\left\|\sum_{i=a_{k}}^{b_{k}} m(A) x_{i}\right\|=\left\|m(A)\left(\sum_{a_{k}}^{b_{k}} x_{i}\right)\right\|>\varepsilon .
$$

Now using the fact that $m$ being $s$-bounded imples that $\tilde{m}$ is regular, we construte a sequence $\left(f_{k}\right)$ of non-negative continuous functions of norm one so that

$$
\left\|L\left(\sum_{i=a_{k}}^{b_{k}} f_{k} x_{i}\right)\right\|>\varepsilon, \quad k=1,2, \ldots
$$

However, by Lemma $2.1 \sum_{k} \sum_{i=a_{k}}^{b_{k}} f_{k} x_{i}$ is w.l.s., and therefore $\sum_{k} \sum_{i} L\left(f_{k} x_{i}\right)$ is u.c., a contradiction. Thus $m(A)$ is u.e.

(v) Next, suppose that $L$ is completely continuous. Thus $m$ is $s$-bounded, and $\mathfrak{a}=\left\{\left|m_{z}\right|: z \in \mathbb{F}_{1}^{*}\right\}$ is conditionally weakly compact. Furthermore, there is $0 \leqslant \lambda \in \operatorname{rea}(\Sigma)$ so that the elements of $\mathfrak{a}$ are uniformly absolutely continuous with respect to $\lambda[9$, Chapter IV]. 
Due to the regularity properties of $m$, it will suffice to show that $m(A)$ is completely continuous for each closed $G \delta$-subset $A$ of $H$. Let $A$ be a closed $G \delta$-set, let $\left(U_{n}\right)$ be a decreasing sequence of open sets so that $\cap U_{n}=A$, and let $\left(f_{n}\right)$ be a sequence of real valued continuous functions so that $f_{n}(A)=1,0 \leqslant f_{n} \leqslant 1$, and support $\left(f_{n}\right) \subset U_{n}, n=1,2, \ldots$ Now suppose that $x_{i} \stackrel{w}{\rightarrow} 0,\left\|x_{i}\right\| \leqslant 1, \delta>0$, and

$$
\left\|m(A) x_{i}\right\|>\delta, \quad i=1,2, \ldots
$$

Letting $\left(z_{i}\right) \subset F_{1}^{*}$ so that $m_{\left(x_{i}, z_{t}\right)}(A)>\delta$ for each $i$ and setting $\mu_{i}=m_{\left(x_{i}, z_{i}\right)}$, we claim that $\mu_{i}\left(f_{n}\right)=\int f_{n} d \mu_{i} \stackrel{n}{\rightarrow} \mu_{i}(A)$ uniformly in $i$. To see this, note that $\left|\mu_{i}\left(f_{n}\right)-\mu_{i}(A)\right| \leqslant$ $\leqslant\left|\mu_{i}\right|\left(U_{n} \backslash A\right)$, and $\left|\mu_{i}\right|\left(U_{n} \backslash A\right) \stackrel{n}{\rightarrow} 0$ uniformly in $i$ since $\lambda\left(U_{n} \backslash A\right) \stackrel{n}{\rightarrow} 0$. Next we clajm that $\mu_{i}\left(f_{n}\right) \stackrel{i}{\rightarrow} 0$ for each $n$. In fact,

$$
\mu_{i}\left(f_{n}\right)=z_{i}\left(\int f_{n} x_{i} d m\right)=z_{i}\left(L\left(f_{n} x_{i}\right)\right)
$$

And since $x_{i} \stackrel{w}{\rightarrow} 0$, clearly $f_{n} x_{i} \stackrel{w}{\rightarrow} 0$; thus

$$
\left|z_{i} L\left(f_{n} x_{i}\right)\right| \leqslant\left\|L\left(f_{n} x_{i}\right)\right\| \rightarrow 0
$$

Next choose $\varepsilon$ so that $0<\varepsilon<\delta / 2$, and let $N$ be a natural number so that $\mid \mu_{i}\left(f_{n}\right)-$ $-\mu_{i}(A) \mid<\varepsilon$ for each $i$ and each $n \geqslant N$. There are infinitely many values of $i$ for which $\left|\mu_{i}\left(f_{N}\right)\right|<\varepsilon$; and, for these same values of $i,\left|\mu_{i}(A)\right|<2 \varepsilon<\delta$, a contradiction. Thus $m(A)$ is c.c.

(vi) Now suppose that $L$ is nuelear. Let $\sum a_{n} \otimes b_{n}$ be an expansion of $L$ as in the definition, and let $y^{*} \in F^{*}$ act on $m(A) x=L^{* *}\left(\xi_{A} \cdot x\right)$. Then

$$
\left(L^{* * *} \xi_{A} \cdot x, y^{*}\right)=\left(\xi_{A} * x, L^{*} y^{*}\right),
$$

and

$$
L^{*} y^{*}=\sum a_{n} \otimes y^{*}\left(b_{n}\right)=\sum y^{*}\left(b_{n}\right) a_{n}
$$

where this sum converges in the norm topology of $C(H, E)^{*}$. Therefore

$$
\xi_{A^{*}} \cdot x\left(\sum y^{*}\left(b_{n}\right) a_{n}\right)=\sum y^{*}\left(b_{n}\right) \xi_{A} \cdot x\left(a_{n}\right)=\sum y^{*}\left(b_{n}\right) \mu_{\left(x, a_{n}\right)}(A),
$$

where $\mu_{\left(x, a_{n}\right)}$ is the regular Borel measure on $\Sigma$ generated by the functional $\left(x, a_{n}\right)$ on $O(H)$. Thus

$$
m(A)(\cdot)=\sum \mu_{\left((\cdot), a_{n}\right)}(A) \otimes b_{n}
$$

is nuclear. 
(vii) For the case when $L: C(H, E) \rightarrow F$ is quasinuclear, we note that by Pietsch $[18,3.2 .6], L: C(H, E) \rightarrow m(S)$ is nuclear where $S=F_{1}^{*}$. Consequently, the quasinuclearity of each $m(A)$ follows from (vi).

(viii). Suppose that $L$ is strictly singular and that $A \in \Sigma$. Further suppose that $m(A)$ is not strictly singular. Then there is an infinite dimensional subspace $M$ of $E$ so that $\left.m(A)\right|_{M}$ has a bounded inverse. Therefore there is a number $\delta>0$ so that $\|m(A) x\| \geqslant \delta\|x\|$ for each $x \in M$.

Now let $\varepsilon>0$ so that $2 \varepsilon<\delta$; and, using the regularity on $\tilde{m}$ (recall that $m$ is $s$-bounded), let $K$ be a nonempty compact set and let $U$ be an open set so that $K \subset A \subset U$ and $\tilde{m}(U \backslash K)<\varepsilon$. Thus if $q$ is a non-zero member of $O(H, E)$ so that $q$ vanishes outside of $U,\|q\|=1$, and $q(K)=x$, where $\|x\|=1$, then

$$
\begin{aligned}
\|L(q)\| & =\left\|\int_{\sigma} q d m\right\| \geqq\left\|\int_{K} q d m\right\|-\left\|\int_{\widetilde{K}} q d m\right\| \geqslant\left\|\int_{K} q d m\right\|-\tilde{m}(U \backslash K) \\
& \geqslant\|m(K) x\|-\varepsilon \geqslant\|m(A) x\|-\|m(A \backslash K) x\|-\varepsilon \\
& \geqslant\|m(A) x\|-\tilde{m}(A \backslash K)-\varepsilon \geqslant(\delta-2 \varepsilon)\|x\| .
\end{aligned}
$$

Then let $f \in C(H)$ so that $\|f\|=1, f(K)=1$, and $f(H \backslash U)=0$. Set $B=\{f \cdot x$ : $x \in M\}$. Clearly $B$ is an infinite dimensional subspace of $C(H, E)$; and, from the inequalities in the preceing paragraph, it follows that $L_{B}$ is invertible, a contradiction.

\section{5. - The $\delta_{m}$-topology and a spectral result.}

Suppose that $m \leftrightarrow L$ is a representing measure and $A \in \Sigma$. For $z \in F^{*}$, we define $\delta_{(m, A)}(z)$ to be $\left|m_{x}\right|(A)$. Then $\left\{\delta_{(m, A)} A \in \Sigma\right\}$ defines a locally convex topology on $F^{*}$ which we denote by $\delta_{m}$; in fact, $\delta_{m}$ is a semimetric topology. We denote the collection of all compact $G_{\delta}$ subsets of $H$ by $\mathscr{H}$, and $\delta_{m}^{\prime}$ will denote the topology on $F^{*}$ defined by $\left\{\delta_{(m, A)}: A \in \mathcal{M}\right\}$. In this section, we briefly study certain relationships between $\delta_{m}^{\prime}$ and the operator $L$. In particular, we obtain a spectral result for $L: C_{0}(H, E) \rightarrow C_{0}(H, E)$, where $E$ is special type of space which does not contain an isomorph of $c_{0}, H$ is locally compact, and $C_{0}(H, E)$ are those continuous functions vanishing at $\infty$. The topology $\delta_{m}$ was studied in [14] and [4]; this study was extended to $L^{p-s p a c e s ~ i n ~}[1]$.

5.1 Definimron. - A $B$-space $E$ is said to have the Schur property if every weak Cauchy sequence in $E$ is norm convergent.

For the general considerations on spectral theory used is the following discussion, we refer the reader to DUNFord and SOHWARTz [9, Chapter VII] and BACHMAN and NARICI [3, Chapter 21]. In particular, for the Fredholm Alternative we refer to [3]. In this section, we assume the scalar field is $C$, the complex numbers. If $T$ is an operator, then $R(T)$ will denote the image of $T$. 
5.2 Theorem. - Let $E$ be a $B$-space with the Sehur property and suppose that $x: O_{0}(H, E) \rightarrow O_{0}(H, E)$ is weakly compact. If $\lambda \in C, \lambda \neq 0$, and $m \leftrightarrow \lambda-L$, then the following are equivalent:

(i) $\lambda \in \varrho(L)(=$ resolvent set of $L)$;

(ii) $\left(C_{0}(H, E)_{1}^{*}, \delta_{m}^{\prime}\right)$ is a Hausdorff space;

(iii) $\delta_{m}^{\prime} \geqslant w^{*}$ on $O_{0}(H . E)_{1}^{*}$.

Proof. - (iii) $\Rightarrow$ (ii). Suppose that $\delta_{m}^{\prime} \geqslant w^{*}$ on $C_{0}(H, E)_{1}^{*}$ and let $0 \neq z \in C_{0}(H, E)_{1}^{*}$. Therefore $\left|m_{z}\right|(H)>0$. But $\left|m_{z}\right|$ is regular and consequently there is a compact $G_{\delta}$, say $K$, so that $\left|m_{z}\right|(K)>0$.

(ii) $\Rightarrow$ (iii). Since $\delta_{m}^{\prime}$ is a Hausdorff topology, then $\left|m_{z}\right|(H)=\left\|(\lambda-L)^{*} z\right\|>0$ for $0 \neq z$. But $(\lambda-I)^{*}$ being injective implies that $\lambda-I$ has dense range.

Now suppose that $z_{\alpha} \stackrel{\delta_{m}^{\prime}}{\rightarrow} z$ in $C_{0}(H, E)_{1}^{*}$ and let $y \in O_{0}(H, E)$. We claim that $z_{\alpha}(y) \rightarrow z(y)$. Suppose not. Then, without loss of generality, we may assume the existence of a positive number $\varepsilon$ so that $\left|z_{\alpha}(y)-z(y)\right|>\varepsilon$ for each $\alpha$. Choose $f \in C_{0}(H, E)$ so that $\left\|\int_{A} f d m-y\right\|<\varepsilon / 4$, and pick $\alpha_{0}$ so that $\alpha \geqslant \alpha_{0}$ implies that $|m|_{z_{\alpha}-z}(A)<\varepsilon /(\|f\|+1) 4$ where $A$ is a compact $G_{\delta}$. Then, for $\alpha \geqslant \alpha_{0}$ we have

$$
\begin{aligned}
& \left|\left(\int_{A} f d m, z_{\alpha}-z\right)\right| \\
& =\left|\left(\int f d m-y, z_{\alpha}-z\right)+\left(y, z_{\alpha}-z\right)\right| \\
& \quad \geqslant\left|\left(y, z_{\alpha}-z\right)\right|-\left|\left(\int_{A} f d m-y, z_{\alpha}-z\right)\right| \\
& \quad \geqslant\left|\left(y, z_{\alpha}-z\right)\right|-2|| \int_{A} f d m-y||>\varepsilon / 2 .
\end{aligned}
$$

But

$$
\begin{aligned}
\left|\left(\int_{A} f d m, z_{\alpha}-z\right)\right| & \leqslant\|f\||m|_{\left(z_{\alpha}-z\right)}(A) \\
& \leqslant\|f\| \varepsilon / 4(\|f\|+1),
\end{aligned}
$$

and an obvious contradiction is achieved. The implication follows.

(i) $\Rightarrow$ (ii). Suppose $\lambda \in \varrho(K)$. Then $\lambda-L$ is invertible; hence $R(\lambda-L)=O_{0}(H, E)$. As a result of this, it follows that $(\lambda-L)^{*}$ is injective, and thus $\delta_{m}^{\prime}$ is a $T_{\mathbf{2}}$ topology.

(ii) $\Rightarrow$ (i). Suppose that $\delta_{m}^{\prime}$ is a Hausdorff topology. Then, if $z \neq 0$,

$$
\left|m_{z}\right|(H)=\left\|(\lambda-L)^{*} z\right\|>0 \text {. }
$$

Therefore $R(\lambda-L)$ is dense in $C_{0}(H, E)$ since $(\lambda-L)^{*}$ is injective. But, by Theo- 
rem 5.5 in Brooks and Lewis [4], $L^{2}$ is compact, and consequently $R(\lambda-L)$ is closed, i.e.

$$
R(\lambda-L)=C_{0}(H, E)
$$

By the results in 21A.2 of BACHMAN and NARICI [3], this implies that $\lambda-L$ is bijective; by the Open Mapping Theorem, $\lambda \in \varrho(L)$.

For our final result, we need the following definition.

5.3 Definition. - Suppose that $n \leftrightarrow T, m \leftrightarrow L$. We say that $n$ is strongly absolutely continuous with respect to $m(n \lll m)$ provided that for each $A \in \Sigma$ and $x \in E_{1}$

$$
n(A) x \in \overline{\bigcup\left\{m\left(A_{i}\right) x_{i}: x_{i} \in E_{1}\right\}} .
$$

We write $T \lll L$ to indicate that $n \lll m$.

5.4 Theorem. - Suppose that each of $L$ and $T$ is an operator from $C_{0}(H, E)$ to $F, n \leftrightarrow T, m \leftrightarrow L$.

(i) If $T$ is compact, $L \lll T$, and $L$ has dense range, then $T$ has dense range, $L$ is compact, and $\delta_{n}^{\prime}=\delta_{m}^{\prime}=w^{*}$ on $F_{1}^{*}$.

(ii) Conversely, if $\left(F_{1}^{*}, \delta_{n}^{\prime}\right) \approx\left(F_{1}^{*}, \delta_{m}^{\prime}\right)$, then $\overline{R(L)}=\overline{R(T)}$.

(iii) Suppose that $E$ has the Schur property, $F=O_{0}(H, E), \lambda \neq 0, T$ is weakly compact, $\lambda-L \ll \lambda-T$, and $\lambda \in \varrho(L)$. Then $\lambda \in \varrho(T)$.

Proof. (i) From Lemma of 2 Lewis [14], $\overline{L\left(O_{0}(H, E)_{1}\right)} \subset T\left(C_{0}(H, E)_{1}\right)$ and therefore $R(T)$ must be dense and $L$ must be compact. So consequently, by Theorem 4.5 of Brooks and Lewis [4], $\delta_{n}^{\prime}=w^{*}=\delta_{m}^{\prime}$ on $F_{1}^{*}$.

(ii) Since $\left(F_{1}^{*}, \delta_{m}^{\prime}\right)$ is homeomorphic to $\left(F_{1}^{*}, \delta_{n}^{\prime}\right)$, then $\left\|L^{*}(z)\right\|=0$ if and only if $\left\|T^{*}(z)\right\|=0$. But $z \in \operatorname{ker}\left(L^{*}\right)$ if and only if $z$ kills $R(L)$, i.e. $z \in R(L)^{\perp}=\overline{R(L)^{\perp}}$. Therefore $\overline{R(L)^{\perp}}=\overline{R(T)^{\perp}}$; and their respective orthogonal complements in $F$,

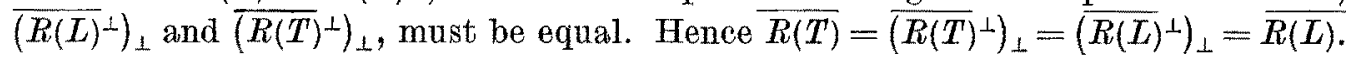

(iii) Since $\lambda-L$ is invertible, $R(\lambda-L)=C(H, E)$; and, by Lemma 2 of Lewis [14], $R(\lambda-L) \subset \overline{R(\lambda-T)}$. But by the Fredholm Alternative construction in BadHMar and NARICI [3], $R(\lambda-T)=\overline{R(\lambda-T)}$. Thus $\lambda-T$ is a surjective operator, and by [3] again, $\lambda \in \varrho(T)$.

\section{REFERENCES}

[1] R. Axò - A. DE KoRvin - L. E. Kunes, Topologieal aspeets of q-regular measures, Studia Math., 48 (1973), pp. 49-60.

[2] B. D. Anderson, Projections and extension maps in $O(T)$, Ill. J. Math., 17 (1973), pp. 513-517. 
[3] G. BaChMaN - L. Nanior, Funotional Analysis, Academic Press, New York (1968).

[4] J. Brooks - P. Lewis, Linear operators and vector measures, Trans. Amer. Math. Soc., 192 (1974), pp. 139.162.

[5] J. Brooks - P. Lewrs, Linear operators and vector measures, II, Math. Z., 144 (1975), pp. 45-53.

[6] E. W. Cheney - K. H. Price, Minimal projections, Approximation theory, Proc. Sympos., Lancaster (1969), pp. 261-289, Aeademic Press.

[7] J. Drestex - B. Fatres, On vector measures, Trans. Amer. Math. Soc., 198 (1974), pp. 253-271.

[8] N. Dinculand, Vector measures, Pergamon Press, Berlin (1967).

[9] N. DunFord - J. SchwarTz, Linear operators, Part I, Interscience, New York (1958).

[10] S. Goldberg, On Sobcayle's projeetion theorem, Am. Math. Month., 76 (1969), pp. 523.526.

[11] J. Howard, The comparison of an unconditionally converging operator, Studia Math., 33 (1969), pp. 295-298.

[12] G. Koтне, Über eimen Satz von Sobezyk, Anals da Faculdade de Ciencias da Porta $3^{\circ}$ e $4^{\circ}, 49$ (1966), pp. 1-6.

[13] P. LAwIs, Some regularity conditions on vector measures with finite semivariation, Rev. Roumaine Math., 15 (1970), pp. 375-384.

[14] P. Lewı, Permanence properties of absolute continuity conditions, International Conference on Vector and Operator Valued Measures and Applications, Snowbird, Utah, Academic Press (1973), pp. 197-206.

[15] A. PelczYNSK, Banach spaces on which every unconditionally converging operator is weakly compact, Bull. Acad. Polon. Sci., serie sci., math., astr. et phys., 10 (1962), pp. 641-648.

[16] A. PeLCZYNsKr, On striotly singular and strictly cosingular operators - I: Striotly singular and strictly cosingular operators in $O(S)$-spaces, Bull. Acad. Polon. Sci., serie sci., math., astr. et phys, 13 (1965), pp. 31-36.

[17] A. Pietsch, Absolut p-summierende Abbildungen in normierten Raumen, Studia Math., 28 (1967), pp. 333-353.

[18] A. Pretsch, Nuclear locally convex spaces, Ergeb. Math. Grenz., vol. 66, Springer-Verlag New York (1972).

[19] H. RosenTHAT, On relatively disjoint families of measures, with some applications to Banach space theory, Studia Math., 27 (1970), pp. 13-36.

[20] A. SoвczYK, Projections of the space $m$ on its subspace $c_{0}$, Bull. Amer. Math. Soe., 47 (1941), pp. 938-947.

[21] C. SWARTz, Uneonditionally converging operators on the space of continuous funetions, Rev. Roumaine Math., 17 (1972), pp. 1695-1702.

[22] C. SWARTz, Absolutely summing and dominated operators on spaces of vector-valued continuous functions, Trans. Amer. Math. Soe., 179 (1973), pp. 123-132.

[23] W. A. VEech, Short proof of Sobczyk's theorem, Proc. Amer. Math. Soc., 28 (1971), pp. 627-628. 